\title{
VORTEX SHEDDING FREQUENCY OF POROUS CIRCULAR TUBES WITH VARYING POROUS PROPERTIES ALONG THE CIRCUMFERENCE
}

\author{
U. JANOSKE \\ University of Wuppertal, Germany.
}

\begin{abstract}
A numerical study on the laminar vortex shedding frequency on a porous and partially porous circular tube has been made. Porous tubes characterised by an inner and outer diameter of the porous zone are subjected to a uniform flow. The porous region is divided in eight segments of $45^{\circ}$ with a different porous structure. The porous behaviour has been modelled by a Darcy-equation. To ensure a laminar vortex shedding, the Reynolds number has been chosen between 40 and 200. The influence of the Reynolds number and the properties of the porous zone (thickness, pressure drop), characterised by a dimensionless thickness $\Lambda$ and a Darcy number $D a$ on vortex shedding frequency (dimensionless Strouhal number $\mathrm{Sr}$ ) and volume flow through the porous zone (dimensionless volume flow $\Pi$ ) has been analysed.
\end{abstract}

Keywords: CFD, cylinder, frequency, porous, segment, vortex shedding.

\section{INTRODUCTION}

The separation of flows around bluff bodies has been studied over the last decades in many different ways. Especially, the flow around cylinders was subject of numerous publications, e.g. Qu et al. [1]. In most cases, these separations have a negative influence on the acoustics as well as the generation of structural vibrations. A lot of effort has been made to control the vortex shedding by several means like splitter plates, deflectors, rings, combinations of cylinders in tandem, etc. Another interesting method for a passive control of vortex shedding is the use of a porous sheath around the cylinder. Bruneau and Mortazavi [2] reported on a massive reduction of flow-induced vibrations for several geometries (pipe, square). Experiments for foam-wrapped cylinders have been reported by Abdi et al. [3]. For Reynolds numbers between 1500 and 8000, the foam-wrapped cylinder increased the wake size compared to solid cylinders. Bhattacharyya and Singh [4] made an extensive study for a laminar vortex separation up to a Reynolds number of 250 for a cylinder covered by a porous sheath. The porous sheath leads to a damping of the separation of the flow, i.e. lower Strouhal numbers. They also found that non-linear effects can be neglected in the porous zone. Sobera et al. [5] considered cylinders surrounded by a porous material with a small distance in between for a constant Reynolds number of 3900. Using a direct numerical simulation (DNS), they observed a laminar and periodic flow between the porous layer and the solid cylinder. Bhattacharyya et al. [6] considered the fluid flow as well as a solute transport around and through a porous cylinder. They used a Reynolds number between 1 and 40 for varying pressure losses in the porous zone, described by the dimensionless Darcy numbers (Da). The numerical simulations showed a shift of the point of separation for small Darcy numbers to the rear stagnation point. The flow through a porous object with changing porous properties has not been reported yet in literature. Therefore, in this paper, a porous tube which is divided in eight segments in circumferential direction is used to examine the influence of varying porous structures on vortex shedding behaviour. The influence of different combinations on 
the separation of the flow is studied. After the explanation of the simulation model and the numerical method in $\S 2$, the numerical results will be presented in $\S 3$.

\section{NUMERICAL METHOD}

In the next section, the geometry of the simulation model, the assumptions as well as the governing equations will be presented.

\subsection{Geometry}

Figure 1 shows the geometry used for the simulations. The porous tube with the outer and inner radius $r_{\mathrm{o}}$ resp. $r_{i}$ is segmented into eight different regions, i.e. $45^{\circ}$, in circumferential direction. Each segment is allowed to have a different porous structure. The tube is subjected to a flow with a uniform constant velocity $u_{0}$ in positive $x$-direction. The vortex frequencies have been determined at three different locations $x_{\mathrm{p} 1}, x_{p 2}$ and $x_{p 3}$, which have a distance of $\frac{x_{p 1}}{r_{o}}=28.6, \frac{x_{p 2}}{r_{o}}=57.2, \frac{x_{p 3}}{r_{o}}=85.8$ from the center of the porous tube and are located on the $\mathrm{x}$-axis, by evaluating the velocity fluctuations. The angular position is given by the angle $\Theta$ which is measured beginning from the negative $x$-axis.

The computational domain covers a distance of 171.43 times the radius $r_{\mathrm{o}}$ in flow direction and 28.57 times the radius $r_{\mathrm{o}}$ perpendicular to the flow direction. The reference length used in this study is $r_{\mathrm{o}}=3.5 \mathrm{~mm}$.

\subsection{Assumptions}

To reduce the computational effort several assumptions have been made:

- The flow is assumed to be incompressible, isothermal and laminar with constant material properties for dynamic viscosity $\mu$ and density $\rho$.

- The flow will be modelled in two dimensions, i.e. the flow in $z$-direction will not be considered.

- The porous material with the porosity $\epsilon$ will be modelled as a homogeneous porous media in the CFD simulations using a Darcy-Forchheimer model for the pressure drop calcula-

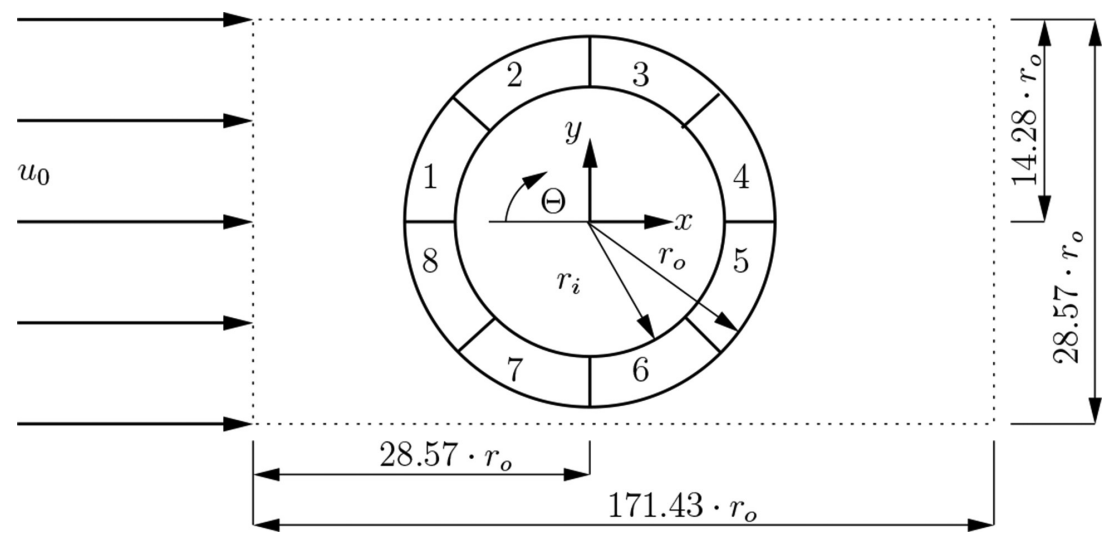

Figure 1: Schematic drawing of the simulation model. 
tion assuming highly porous systems. Due to the small velocities in the porous media, the Darcy term is assumed to be the dominating one.

- In order to reduce the number of simulations, the values for the porous structure are assumed to be symmetric to the $x$-axis, i.e. segments 1 and 8, 2 and 7, 3 and 6, 4 and 5 will have equal properties and are combined to the zones I, II, III and IV.

\subsection{Governing equations}

The flow conditions, i.e. velocity vector $\mathrm{u}$ respectively adapted velocity $\mathrm{q}=\epsilon^{\bullet} \mathrm{u}$ and pressure $\mathrm{p}$ can be calculated by the continuity (eqn (1)) and the momentum balance (eqn (2)) including the source term $s_{p}$ to take into account the pressure drop of the porous zone. The porous-solid, porous-porous and the fluid-porous interface are modelled with a unified single-domain approach according to Bhattacharyya and Singh [4]. The pressure drop is calculated with the equation given by eqn (3) using the Darcy coefficient $d\left[1 / \mathrm{m}^{2}\right]$ and the Forchheimer coefficient $\mathrm{c}_{F}$. By applying one set of equations with switching terms for the porosity, the interfacial conditions are automatically considered. Furthermore, the porosity is assumed to be highly porous $(\in \rightarrow 1)$ and due to the small velocities the Forchheimer term $\left(c_{\mathrm{F}}=0\right)$ is neglected. The equations are solved using the Open Source CFD-code Open-FOAM ${ }^{\circledR}$ version 2.3. For the calculation of the pressure field, a PIMPLE (merged PISO-SIMPLE) algorithm is applied. For the temporal discretisation an implicit Crank-Nicolson scheme with an adaptive time step (Courant numbers smaller 0.5) and for the spatial discretisation, a second-order linear scheme is used.

$$
\begin{gathered}
\nabla \cdot \mathrm{q}=0 \\
\frac{\rho}{\epsilon} \frac{\partial q}{\partial t}+\frac{\rho}{\epsilon^{2}} \cdot \nabla \cdot(\mathrm{qq})=\mu \nabla \cdot\left[\nabla \mathrm{q}+(\nabla \mathrm{q})^{T}\right]-\nabla p+\mathrm{Sp} \\
S p=-\mu d q-C_{F} \sqrt{d}|\mathrm{q}| \mathrm{q}
\end{gathered}
$$

The equations are solved assuming a constant velocity and a zero gradient for the pressure at the inflow boundary on the left side of the computational domain in Fig. 1. At the outflow, a fixed pressure $(p=0)$ and a zero gradient boundary condition for the velocity has been used. The upper and lower boundaries are slip boundary conditions, applying a zero gradient boundary condition for velocity and pressure. In the simulations for a solid cylinder which are used as a reference, the wall of the cylinder has no-slip boundary conditions $(u=0)$ for the velocity and a zero gradient for the pressure.

\subsection{Dimensionless numbers}

The problem can be described by a set of dimensionless numbers. The Reynolds number Re will be defined by the outer radius $r_{0}$, the uniform velocity $u_{0}$ and the material properties of the fluid.

$$
R e=\frac{2 \rho r_{0} u_{0}}{\mu}
$$

The dimensionless thickness of the tube $\Lambda$ is given by the inner and outer radius $r_{i}$ respectively $r_{0}$, respectively. In the simulations, the outer radius has been kept fix and the inner radius was varied to adjust different ratios of $\Lambda$. 


$$
\Lambda=\frac{1}{2}\left(1-\frac{r_{i}}{r_{0}}\right) .
$$

The porous zone parameter $d$ is made dimensionless by the outer diameter $r_{\mathrm{o}}$ which gives the dimensionless Darcy number $D a$.

$$
D a=d \cdot r_{o}^{2}
$$

The dimensionless Strouhal number $S r$ gives the dimensionless form of the frequency $f$ of the vortex shedding.

$$
S r=\frac{2 f r_{o}}{u_{o}}
$$

The time-averaged volume flow through the inner flow domain $Q_{\text {porous }}$ bounded by the radius $r_{i}$ is related to the total volume flow $Q_{\text {total }}$ described by the dimensionless volume flow $\Pi$.

$$
\Pi=\frac{Q_{\text {porous }}}{Q_{\text {total }}}
$$

\subsection{Grid-independent study and validation}

Grid-sensitivity studies have been made regarding the overall grid size as well as the ratio of the first grid point to the surface of the cylinder. In all the simulations, a two-dimensional, structured grid has been used. Figure 2 shows the Strouhal number for a solid cylinder and a Reynolds number $R e=140$ as a function of different numbers of control volumina as well as different distances of the first grid point to the cylinder. The grid size was varied between approximately $40 \mathrm{k}$ control volumina up to $1.2 \mathrm{M}$ control volumina showing a grid independency for grids larger than $600 \mathrm{k}$ control volumina. The ratio of the distance of first grid point to the cylinder $\Delta r$ and the radius of the cylinder $r_{\mathrm{o}}$ was varied between $7 \cdot 10^{-4}$ and 0.06 showing a grid independency smaller than 0.02 . A ratio of about $5 \cdot 10^{-3}$ was chosen in the simulations, which is slightly larger than the one recommended by Rajani et al. [7].

\section{RESULTS AND DISCUSSIONS}

Before the discussion of the results for a varying porosity, simulations for a homogeneous porous zone as well as a solid cylinder for validation purposes are described. The Reynolds, Darcy and thickness number have been varied for 36 combinations ranging from $40<\operatorname{Re}<200,1.225 \cdot 10^{-2}<D a<1.225 \cdot 10^{16}, 0.075<\Lambda<0.375$.

Figure 3a shows the Strouhal number $S r$ plotted against the Reynolds number Re for different Darcy numbers $D a$. The thickness value $\Lambda$ shows no difference in the results, i.e. for the Darcy numbers used, the influence of A can be neglected. Higher Reynolds numbers lead to an increase of the Strouhal number, i.e. a higher frequency of the vortex shedding. In Fig. 3a, experimental results by Williamson [8] for a solid cylinder are plotted as well showing a similar increase like the values for a porous tube. The difference between simulation and experimental data is between $3.8 \%$ for $R e=80$ and $1.5 \%$ for $R e=200$ with slightly higher values for the numerical results. 


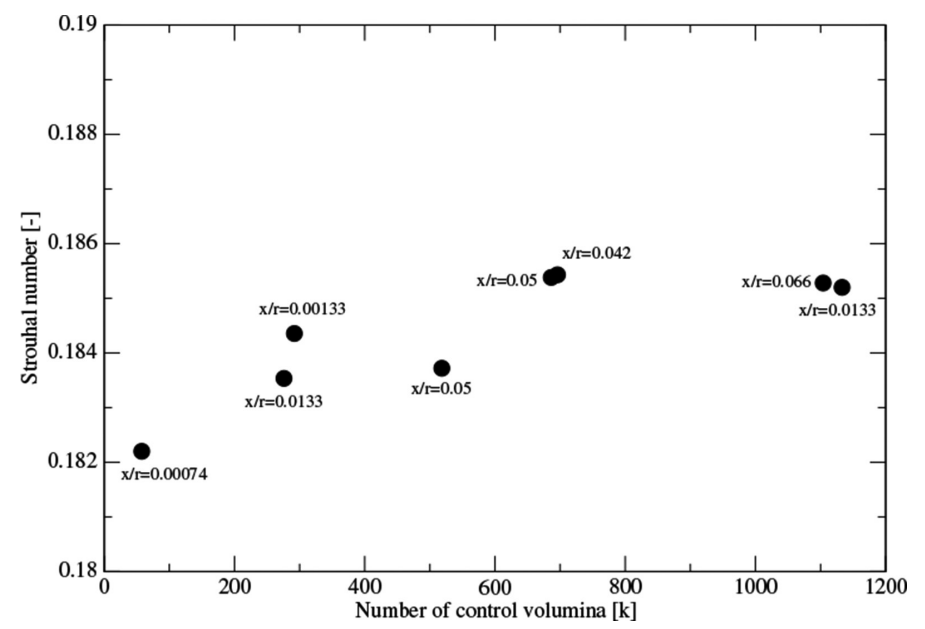

Figure 2: Grid sensitivity study for different grid sizes and distances from the first grid point to the cylinder $(R e=140)$.
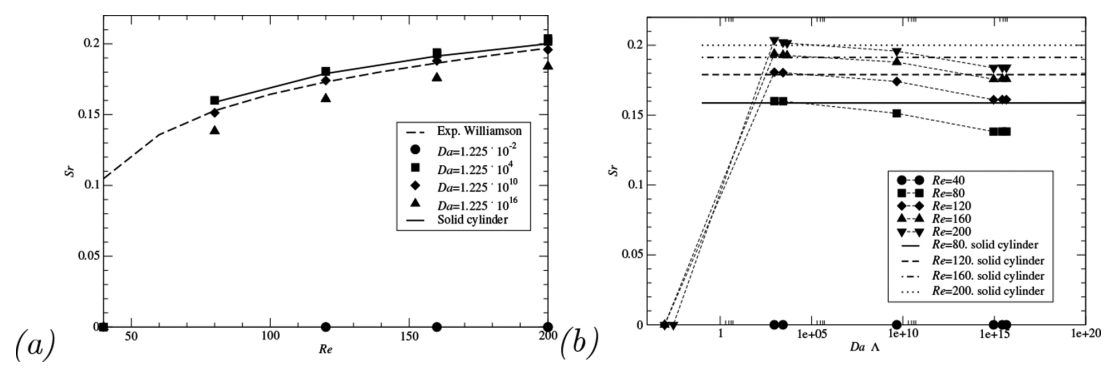

Figure 3: Strouhal number $S r$ as a function of the Reynolds number Re for different Darcy numbers $D a$ and comparison with experiments by [8] (a) and as function of the Reynolds number $R e$ for different combinations of $\Lambda \cdot D a(b)$.

The influence of the Darcy number on the separation frequency can be observed in Fig. $3 b$ showing the Strouhal number $S r$ plotted against the product of Darcy and thickness number. For small Darcy numbers, the flow resistance is also very small leading to a flow through the porous zone without any vortex shedding. An increase of the Darcy number invokes the beginning of the vortex shedding. The Strouhal number for Darcy numbers in the order of $O$ (4) is similar to the one obtained for the solid cylinder, shown as lines in Fig. 3b. A further increase of the Darcy number leads to a slight decrease of the Strouhal number till a constant value can be found for Darcy numbers of order $O(15)$ and higher. This behaviour can be seen for all Reynolds numbers considered in this study. The parameter set used for the simulations as well as the results for the Strouhal number and the dimensionless volume flow is shown in Table 1. Three different values of Darcy numbers have been used and varied by a Design-of-Experiment approach. The Reynolds number has been kept constant $R e=120$ as well as the thickness of the porous zone $\Lambda=0.225$. Figure 4 shows the Strouhal number $S r$ plotted as a function of Darcy numbers $D a_{1}$ and $D a_{2}$ for the different combinations of the remaining Darcy numbers $D a_{3}$ and $D a_{4}$. The contour plots are generated based on the results (see Table 1) and interpolated by radial basis functions. Linear models for a statistical evaluation of the 
data are not capable of describing the non-linear data. Response surface approaches of second order still show a large deviation in the residual plots. From Fig. 4 the following conclusions can be drawn:

Vortex shedding can only be avoided for combinations with a small Darcy number for segment I and segment IV, i.e. if a part of the flow is allowed to pass through the cylinder.

The maximum value for the Strouhal number can be seen, if the first segment I has the maximum value and the following segments II and III the lowest.

The higher the values in all segments, a nearly constant value for the Strouhal number will be the result. For the combination with the highest values of Darcy numbers (case 25 in Table 1), the Strouhal number is $S r=0.1790$ which is very close to the experimental results of Williamson [8] for a solid cylinder $(S r=0.1802)$.

The results for the Strouhal number are very sensitive to changes of the Darcy numbers for segment I and IV. To explain this behaviour, the flow field will be compared for six selected

Table 1: Simulation parameters and results for Strouhal number $\mathrm{Sr}$ and dimensionless volume flow $\Pi$ for segmented porous zones. Three level of values for the Darcy numbers have been chosen which are indicated by symbols: $\nabla: D a=1.225 \cdot 10^{-2}$, O: $D a=3.87 \cdot 10^{2}, \Delta: D a=1.22510^{6}$.

\begin{tabular}{lllllll}
\hline- & $I$ & $I I$ & $I I I$ & $I V$ & $S r[-]$ & $\Pi[\%]$ \\
\hline 1 & $\mathrm{O}$ & $\mathrm{O}$ & $\nabla$ & $\nabla$ & $\mathbf{0 . 1 7 0 8}$ & $\mathbf{1 . 0 7 0 6}$ \\
2 & $\mathrm{O}$ & $\mathrm{O}$ & $\Delta$ & $\nabla$ & 0.1657 & 1.1534 \\
3 & $\mathrm{O}$ & $\mathrm{O}$ & $\nabla$ & $\Delta$ & 0.1835 & 0.5857 \\
4 & $\mathrm{O}$ & $\mathrm{O}$ & $\nabla$ & $\Delta$ & 0.1802 & 0.0616 \\
5 & $\mathrm{O}$ & $\nabla$ & $\mathrm{O}$ & $\nabla$ & $\mathbf{0 . 1 4 7 0}$ & $\mathbf{0 . 9 0 8 8}$ \\
6 & $\mathrm{O}$ & $\Delta$ & $\mathrm{O}$ & $\nabla$ & 0.1710 & 1.1249 \\
7 & $\mathrm{O}$ & $\nabla$ & $\mathrm{O}$ & $\Delta$ & 0.1976 & 0.1957 \\
8 & $\mathrm{O}$ & $\Delta$ & $\mathrm{O}$ & $\Delta$ & 0.1781 & 0.3755 \\
9 & $\nabla$ & $\mathrm{O}$ & $\mathrm{O}$ & $\nabla$ & $\mathbf{0 . 0 0 0 0}$ & $\mathbf{3 . 6 7 1 4}$ \\
10 & $\Delta$ & $\mathrm{O}$ & $\mathrm{O}$ & $\nabla$ & 0.2006 & 0.0516 \\
11 & $\nabla$ & $\mathrm{O}$ & $\mathrm{O}$ & $\Delta$ & 0.1718 & 0.7377 \\
12 & $\Delta$ & $\mathrm{O}$ & $\mathrm{O}$ & $\Delta$ & 0.1921 & 0.0341 \\
13 & $\mathrm{O}$ & $\nabla$ & $\nabla$ & $\mathrm{O}$ & $\mathbf{0 . 2 1 7 2}$ & $\mathbf{0 . 3 2 9 5}$ \\
14 & $\mathrm{O}$ & $\Delta$ & $\nabla$ & $\mathrm{O}$ & 0.1797 & 0.8883 \\
15 & $\mathrm{O}$ & $\nabla$ & $\Delta$ & $\mathrm{O}$ & 0.1842 & 0.5857 \\
16 & $\mathrm{O}$ & $\Delta$ & $\Delta$ & $\mathrm{O}$ & 0.1752 & 0.6471 \\
17 & $\nabla$ & $\mathrm{O}$ & $\nabla$ & $\mathrm{O}$ & $\mathbf{0 . 1 3 1 9}$ & $\mathbf{2 . 4 9 7 0}$ \\
18 & $\Delta$ & $\mathrm{O}$ & $\nabla$ & $\mathrm{O}$ & 0.2030 & 0.0469 \\
19 & $\nabla$ & $\mathrm{O}$ & $\Delta$ & $\mathrm{O}$ & 0.1561 & 1.3252 \\
20 & $\Delta$ & $\mathrm{O}$ & $\Delta$ & $\mathrm{O}$ & 0.1866 & 0.0380 \\
21 & $\nabla$ & $\nabla$ & $\mathrm{O}$ & $\mathrm{O}$ & $\mathbf{0 . 1 5 0 4}$ & $\mathbf{1 . 4 4 3 5}$ \\
22 & $\Delta$ & $\nabla$ & $\mathrm{O}$ & $\mathrm{O}$ & 0.2074 & 0.0055 \\
23 & $\nabla$ & $\Delta$ & $\mathrm{O}$ & $\mathrm{O}$ & 0.1484 & 1.9924 \\
24 & $\Delta$ & $\Delta$ & $\mathrm{O}$ & $\mathrm{O}$ & 0.1836 & 0.0178 \\
25 & $\mathrm{O}$ & $\mathrm{O}$ & $\mathrm{O}$ & $\mathrm{O}$ & 0.1790 & 0.6838 \\
\hline
\end{tabular}




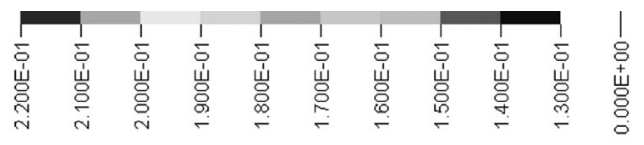
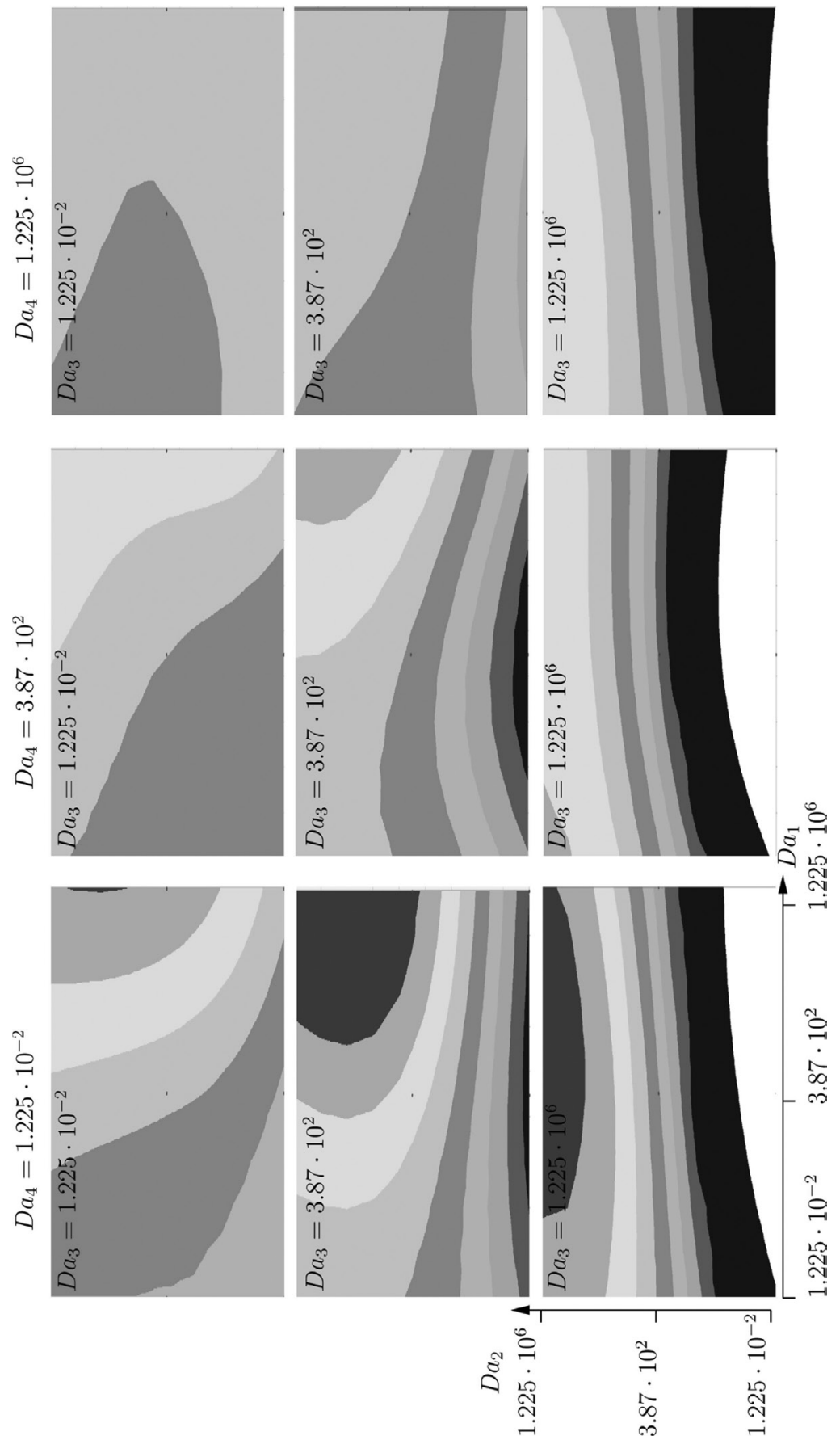

Figure 4: Strouhal number $S r$ as a function of the Darcy numbers for segment $1 D a_{1}$ and segment $2 \mathrm{Da}_{2}$ plotted for combinations of $D a_{3}$ and $\mathrm{Da}_{4}$. 
cases $(1,5,9,13,17$ and 21). In the selected cases, the Darcy number has the values 1.225 . $10^{-2}$ and $3.87 \cdot 10^{2}$ in different arrangements. The values of the Darcy numbers are shown in bold letters in Table 1 for a better overview. For these selected cases, the difference in the normal velocities through outer minus the inner radius of the porous zone have been plotted against the angle $\Theta$, shown in Fig. 5 for a time $t=2.5 \mathrm{~s}$, i.e. positive values indicate a flux into the porous zone and vice versa. The variation shows a strong influence on the Strouhal number ranging from no vortex shedding to the highest Strouhal number $S r=0.2171$. First, these two cases should be discussed: In case 9 with no vortex shedding, the Darcy numbers for segments I and IV, which are positioned normal to the flow direction, have the smallest value. This leads to a flow through the porous zone stabilising the flow. The velocity profile shows between 0 and 90 degree a flow into the porous zone (negative values) and a flow out of the zone between 90 and 180 degree. In case 13 which has the highest Strouhal number observed in this study, the smallest values of the Darcy number can be found for segments II and III which are parallel to the flow direction. This leads to a flow through the porous segments II and III with a volume flow into the porous segment II indicated by the negative flow velocity between 45 and 90 degree. This can be interpreted as a suction into the porous zone. Studies on the Strouhal number for porous cylinders which are subjected to suction and blowing have been reported by Fransson et al. [9]. They found an increase of the Strouhal number for suction and a decrease for blowing. The same phenomena can be observed for this case, where the suction and blowing is only adjusted locally by changing the porous structure. For higher values for the Darcy numbers in segment I and II (case 1), showing a flow around the first part of the cylinder similar to the flow around a solid cylinder, the values of the Strouhal number tend to those of a solid cylinder reported by Williamson [8] $(\mathrm{Sr}=0.1802)$.

Figure 5a shows the comparison of the characteristic cases with no vortex shedding (case 9), the highest values of Strouhal observed (case 13) and comparable Strouhal values to those of solid cylinders (case 21). One can see for the case with no vortex shedding large velocity differences between 0 and 90 degree which means an inflow in the porous region and a negative velocity between 90 and 180 degree. Figure 5 b shows cases 17, 21 and 5 with a Strouhal in the range between 0.1319 and 0.1504 . Although the changes are moderate, the differences in the velocities are large, i.e. a change of the Strouhal number can be obtained by several means.

Figure 6a shows the dimensionless volume flow $\Pi$ plotted against the Strouhal number. The dimensionless volume flow has been obtained by integrating the normal velocities along the inner radius and normalising the value by the inlet volume flow. Smaller Strouhal numbers lead

(a)
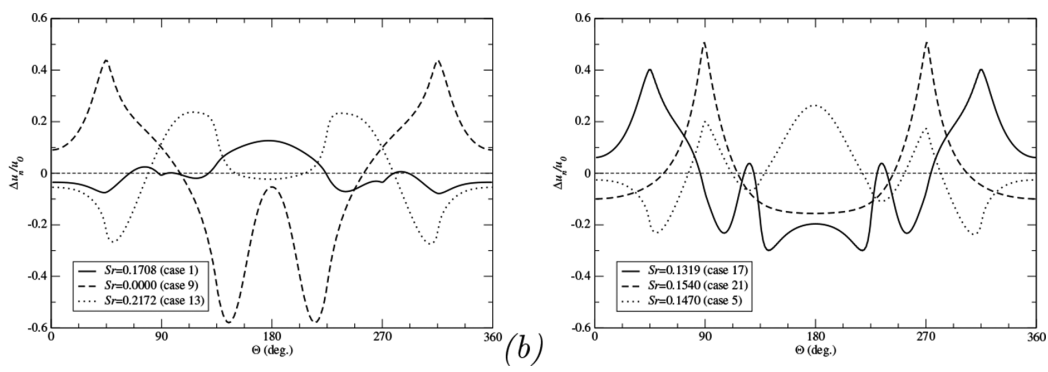

Figure 5: Velocity difference through outer porous zone and inner porous zone plotted against the angle $\Theta$ for cases 1,9 and $13(a)$ and cases 5, 17 and $21(b)$. 

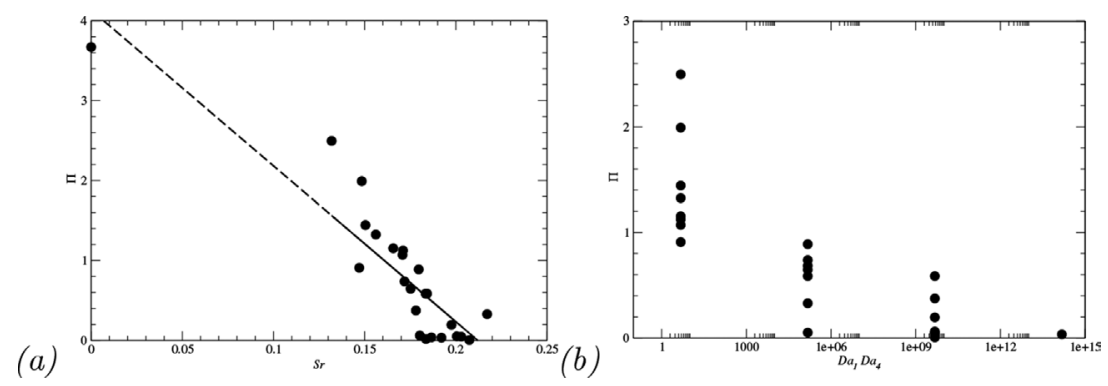

Figure 6: Volume flow ratio $\Pi$ as a function of Strouhal number $\operatorname{Sr}(a)$ and as a function of the product of Darcy numbers $D a_{1}$ and $D a_{4}(b)$.

to a higher volume flow through the porous structure. Despite the deviation of the value from the linear regression curve, the tendency is quite obvious. Smaller Strouhal numbers respectively zero values, if there is no vortex shedding, are observed for smaller Darcy numbers leading to the already mentioned higher volume flow $\Pi$. Figure $6 \mathrm{~b}$ shows the dimensionless volume flow $\Pi$ plotted against a combination of the Darcy numbers $D a_{1}$ and $\mathrm{Da}_{4}$. A tendency can be observed to lower volume flows by increasing the product of the two Darcy numbers. Due to the strong non-linear behaviour of the results, a statistical analysis of the data fails, as already reported. Other combinations (interactions) of Darcy numbers show no effect at all.

\section{CONCLUSIONS}

A numerical model to describe vortex shedding behind tubes with a varying porous structure has been reported. By solving the transient, incompressible Navier-Stokes equations for a two-dimensional model, the vortex separation frequency and the volume flow through the porous zone have been obtained as a function of four different Darcy numbers which describe the pressure drop behaviour of 4 porous segments which span over 45 degrees and are arranged symmetrically to the axis in flow direction. The results show a linear dependency of volume flow through the porous zone on the Strouhal number. The results of the Strouhal number are strongly non-linear, but show a dependency on the values of the Darcy number of the segments normal to the flow direction. By interchanging the Darcy numbers in the different segments, a complete change of the behaviour between no vortex shedding and high separation frequencies can be obtained. The behaviour can be explained by an analysis of the flow field. The results can be used to change the separation behaviour of tubes in flows by a small change of the porous structure and their local value. In further work, the range of parameters $\left(\operatorname{Re}, \Lambda, D a_{i}\right)$ should be extended.

\section{REFERENCES}

[1] Qu, L., Norberg, C., Davidson, L., Peng, S.H. \& Wang, F., Quantitative numerical analysis of flow past a circular cylinder at reynolds number between 50 and 200. Journal of Fluids and Structures, 39, pp. 347-370, 2013. http://dx.doi.org/10.1016/j.jfluidstructs.2013.02.007

[2] Bruneau, C.H. \& Mortazavi, I., Control of vortex shedding around a pipe section using a porous sheath. International Journal of Offshore and Polar Engineering, 16(2), pp. 90-96, 2006.

[3] Abdi, I.A., Hooman, K. \& Khashehchi, M., A comparison between the separated flow structures near the wake of a bare and a foam-covered circular cylinder. Journal of Fluids Engineering, 136(12), pp. 121-203, 2014. 
[4] Bhattacharyya, S. \& Singh, A., Reduction in drag and vortex shedding frequency through porous sheath around a circular cylinder. International Journal for Numerical Methods in Fluids, 65(6), pp. 683-698, 2011.

http://dx.doi.org/10.1002/fld.2210

[5] Sobera, M., Kleijn, C. \& Van den Akker, H., Subcritical flow past a circular cylinder surrounded by a porous layer. Physics of Fluids, 18(3), p. 038106, 2006.

http://dx.doi.org/10.1063/1.2189284

[6] Bhattacharyya, S., Dhinakaran, S. \& Khalili, A., Fluid motion around and through a porous cylinder. Chemical Engineering Science, 61(13), pp. 4451-4461, 2006. http://dx.doi.org/10.1016/j.ces.2006.02.012

[7] Rajani, B., Kandasamy, A. \& Majumdar, S., Numerical simulation of laminar flow past a circular cylinder. Applied Mathematical Modelling, 33(3), pp. 1228-1247, 2009. http://dx.doi.org/10.1016/j.apm.2008.01.017

[8] Williamson, C., Oblique and parallel modes of vortex shedding in the wake of a circular cylinder at low reynolds numbers. Journal of Fluid Mechanics, 206, pp. 579-627, 1989. http://dx.doi.org/10.1017/S0022112089002429

[9] Fransson, J., Konieczny, P. \& Alfredsson, P., Flow around a porous cylinder subject to continuous suction or blowing. Journal of Fluids and Structures, 19(8), pp. 1031-1048, 2004.

http://dx.doi.org/10.1016/j.jfluidstructs.2004.06.005 